\title{
Leucoplasia asociada al hábito de fumar invertido. Presentación de un caso clínico
}

\section{Leukoplakia associated with smoking invested. Clinical case report}

\author{
Medina Márquez $M^{*}$, Carmona Lorduy $M^{* *}$, Álvarez Villadiego $\mathrm{P}^{* * *}$, \\ Díaz Caballero A****
}

\section{RESUMEN}

La mucosa bucal es afectada con frecuencia por agentes exógenos que provocan cambios que se manifiestan como alteraciones hísticas, asociados a riesgos de transformación maligna.

El humo del cigarrillo afecta la mucosa oral notablemente, por lo que es evidente que fumar tiene influencias negativas causadas por la combustión del cigarrillo. Los compuestos carcinógenos contenidos en el humo del cigarrillo son factores que originan daños al fumador entre ellos se destaca lesiones premalignas y malignas. Se presenta el caso de un paciente femenino de 84 años de edad, con hábito de fumar cigarrillo con la candela invertida hace 40 años; Se observó a través de un minucioso examen clínico intraoral múltiples placas blancas y verrugosidades en toda la extensión del paladar, asintomáticas, de tiempo de evolución desconocido y de tamaño variable; teniendo en cuenta lo presentado, se realiza un diagnóstico clínico de leucoplasia, el cual se confirma a través de un estudio anatomopatológico realizado en una muestra que se obtuvo por biopsia incisional en zona del paladar del lado derecho; el informe histológico reporta mucosa palatina con marcada queratosis sin displasia. Se recomendó a la paciente suspender el hábito de fumar insistiendo en la importancia de la prevención de la transformación maligna de la patología y promocionando hábitos saludables. Las lesiones palatinas se le realizaron controles mensuales durante 8 meses.

Palabras clave: Leucoplasia, hábito de fumar, lesiones orales.

\section{SUMMARY}

The oral mucosa is frequently affected by exogenous agents that cause changes that manifest as alterations hísticas, risks associated with malignant transformation.

Cigarette smoke significantly affects the oral mucosa, and it is obvious that smoke has adverse influences caused by the combustion of the cigarette. Carcinogenic compounds contained in cigarette smoke are factors which cause damage to the smoker highlights including premalignant and malignant lesions.

We present the case of female patient aged 84, with cigarette smoking the inverted candle 40 years ago was found by a thorough clinical examination intraoral verrugosidades multiple white plaques on the entire length of palate, asymptomatic evolution time unknown and variable size considering it presented, performing a clinical diagnosis of leukoplakia, which is confirmed by a pathological study conducted in a sample that was obtained by incisional biopsy side palate area law, the report histological reports palatal mucosa with marked keratosis without dysplasia. It is recommended that the patient stop smoking stress the importance of prevention

* Odontóloga. Residente de Estomatología y Cirugía Oral Universidad de Cartagena.

** Odontóloga. Universidad de Cartagena. Especialista en Estomatología y Cirugía Oral. Magíster en Educación. Profesor titular de la Universidad de Cartagena.

*** Odontóloga. Universidad de Cartagena. Especialista en estomatología y cirugía oral. Magíster en Bioquímica clínica. Profesor titular de la Universidad de Cartagena.

**** Odontólogo Universidad de Cartagena. Especialista en Periodoncia Universidad Javeriana. Magíster en Educación Universidad del Norte. Candidato a Doctorado en Ciencias Biomédica. Profesor titular de la Universidad de Cartagena. 
of malignant transformation of pathology and promoting healthy habits. Palatal lesions were performed monthly monitoring for 8 months.

Key words: Leukoplakia, cigarette smoking, oral lesions.

Fecha de recepción: 15 de junio de 2014.

Aceptado para publicación: 26 de noviembre de 2014.

Medina Márquez M, Carmona Lorduy M, Álvarez Villadiego P, Díaz Caballero A. Leucoplasia asociada al hábito de fumar invertido. Presentación de un caso clínico. Av. Odontoestomatol 2015; 31 (4): 261-266.

\section{INTRODUCCIÓN}

La leucoplasia oral (LO) es definida según la organización mundial de salud (OMS) como un parche o placa blanca que no puede ser caracterizada clínica o patológicamente como cualquier otra enfermedad; (1) siendo definida desde hace algunos años, desde un contexto eminentemente clínico, como una lesión predominantemente blanca de la mucosa oral en la que existe un riesgo latente de desarrollar cáncer oral por ser una lesión premaligna (2).

Varios estudios de diferentes países sobre la distribución por edades de la leucoplasia oral tienen en cuenta que es dominante en los individuos de 40-60 años (3). En los últimos años, se ha descrito la relación de la leucoplasia con el habito de fumar de manera frecuente, y se describe como un agente etiológico destacable en la aparición de ésta. Sin embargo, en alguna leucoplasia orales, no es posible encontrar factor etiológico alguno; a ellas se les denominan leucoplasias idiopáticas (2). Otras investigaciones realizadas a pobladores indígenas que mastican tabaco y retienen humo, guardando una relación con el género y los hábitos del tabaco, la franja de las edades en que se presenta esta patología oscila entre los 30 y 50 años. Estos datos sugieren que la ocurrencia de leucoplasia oral puede depender del país de origen, la naturaleza de la población objeto de la investigación y el patrón de utilización del tabaco (3).

El consumo de tabaco y el tabaquismo es uno de las causas más comunes de mortalidad y morbilidad en los países desarrollados y en países en vías de desarrollo por los compuestos carcinógenos contenidos en el humo del cigarrillo como son: policíclicos, hidrocarburos aromáticos, aldehídos arsénicos, níquel y cadmio; afectan la cavidad oral principalmente. Hoy se ha convertido el hábito de fumar en la forma dominante en la mayoría de países; lo que es evidente es que fumar tiene muchas influencias negativas en la cavidad oral, por ejemplo: en la tinción de los dientes y las restauraciones dentales, alteración en la cicatrización de las herida, reducción de la capacidad de olfato, del gusto, en el desarrollo de las enfermedades orales tales como el cáncer oral, la periodontitis, la melanosis del fumador, la lengua vellosa, la leucoplasia y la candidiasis oral (4).

Las causas de las lesiones premalignas y malignas en la cavidad bucal son multifactoriales, dentro de éstas se encuentran los efectos combinados de factores predisponentes y causales exógenos (5).

La leucoplasia se presenta clínicamente como una placa blanca, afectando cualquier zona de la mucosa bucal; el tamaño es variable, desde un $1 \mathrm{~cm}$ de diámetro hasta llegar a ocupar gran área de la mucosa, la superficie puede ser lisa o rugosa, el color no siempre es blanco también se presenta de color gris, amarillenta o en varios colores en una lesión y, generalmente, es asintomática. La (LO) se clasifica en homogéneas y no homogéneas, esta última en eritroleucoplasia, nodulares y exofítica (6). Las homogéneas son lesiones principalmente blancas, uniformes, delgadas, que pueden tener una superficie lisa o arrugada que, en algunos casos, presenta surcos pocos profundos. La no homogénea es una lesión predominantemente blanca que está acompañada de zonas rojas y puede presentar una superficie irregular, nodular y exofítica (2). 
Según el criterio histopatológico, la leucoplasia puede ser sin displasia o con displasia. Éstas se clasifican en: displasia leve, moderada o severa. En la displasia leve, los cambios displásicos son mínimos y quedan confinados al tercio inferior del epitelio; en la moderada los cambios displásicos abarcan los 2/3 inferiores del epitelio y, en la severa, equivale al carcinoma in situ. Los cambios displásicos ocupan más de $2 / 3$, pero no toda la totalidad del epitelio (6).

A pesar de la baja prevalencia, de los diferentes tipos de leucoplasia como son: eritroplasia oral, leucoplasia homogénea y leucoplasia moteada, muestran alteraciones histopatológicas que varían desde displasia epitelial a carcinoma invasor. Estas lesiones deben incluirse entre las lesiones orales con el más alto potencial de transformación maligna (7). La prevalencia de la transformación maligna de la leucoplasia oral varía desde $0,13 \%$ hasta $17,5 \%$ con períodos de observación de 1 a 30 años (3).

El hábito de fumar de manera inversa es una específica y peculiar costumbre en los grupos con bajos recursos económicos, además de que se presenta en las zonas cálidas o tropicales, con mayor frecuencia en las mujeres, especialmente después de la tercera década de la vida. Reportes de estudios, como el de Martínez y Carmona (2000), muestran la gran prevalencia de pacientes que mantienen por años la práctica diaria de fumar de manera invertida, evidenciando un alto porcentaje en la población femenina; la mayoría procedían del área rural y el sitio intraoral más afectado fue la mucosa palatina (8). También hay que mencionar que el aspecto clínico de la mucosa oral en pacientes con el hábito de fumar invertido varía cuando se compara con fumadores convencionales y las zonas más comúnmente afectadas son la lengua y el paladar. La temperatura más alta en prácticas del cigarrillo puede llegar a $760^{\circ} \mathrm{C}$ y el aire intraoral puede ser calentado a $120^{\circ} \mathrm{C}$. Este aumento de la temperatura y los productos de combustión ocasionan la frecuencia de las lesiones dentro de la boca en comparación con fumadores convencionales (9).

Para realizar el seguimiento de la lesión y estudios epidemiológicos de la misma es interesante considerar la denominada clasificación LCP, que busca estandarizar todas las clasificaciones de la LO, propuesta por Axel T, Pimdborg et al en 1997. Clasifica- ción LCP: L (tamaño), C (tipo clínico), P (tipo histológico); L1: Menor de 2 cm; L2: De 2 a 4 cm; L3: Mayor de $4 \mathrm{~cm}$; Lx: No especificado; C1: Leve homogéneo; C2: No homogéneo; C3: No especifica; P1: Sin displasia; P2: Displasia leve; P3: Displasia moderada; P4: Displasia severa; P5: No específico (10).

Es relevante hacer un examen clínico exhaustivo que nos permita hacer detección y prevención en lesiones orales que tienen el riesgo de premalignidad a mediano y largo plazo según sea la persistencia del factor de riesgo y/o la agresión a la cavidad oral.

\section{REPORTE DE UN CASO CLÍNICO}

Paciente femenina de 84 años de edad que es remitida al servicio de Estomatología y Cirugía Oral de la Universidad de Cartagena por presentar molestia en cavidad oral. Al examen clínico se observan múltiples placas, manchas y verrugosidades distribuidas en forma irregular de todo el paladar duro comprometiendo tercio anterior y medio, del lado izquierdo y derecho; con una coloración de tonalidad variante desde un blanco grisáceo hasta un blanco amarillo; tamaño que oscila aproximadamente sobre $3 \times 5 \mathrm{~cm}$, forma variada de superficie verrugosa y consistencia blanda, bordes definidos, asintomática (Fig. 1); no

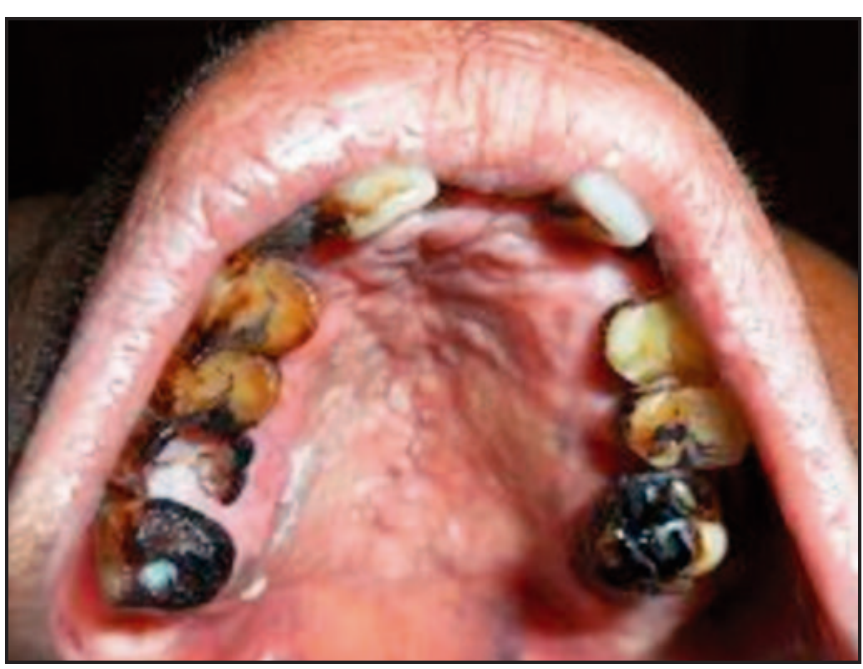

Fig. 1. Imagen fotográfica del maxilar superior donde se aprecia marcada leucoplasia del lado derecho donde se observa lesión verrugosa de color blanco grisáceo, tamaño variable y no desprenden al raspado. 
desprende al raspado e imperceptible por la misma. La paciente reporta ser fumadora invertida desde hace 40 años y refiere fumar 5 cigarrillos al día. En esta paciente se da una impresión clínica de leucoplasia homogénea y, para realizar el diagnóstico definitivo, se programó biopsia incisional.

\section{PROCEDIMIENTO}

Previo consentimiento informado, se aplica técnica anestésica nasopalatina y palatina anterior bilateral, utilizando lidocaína con epinefrina al $2 \%$; luego se realizó incisión romboidal con hoja de bisturí número 15 en borde anterior de paladar duro del lado derecho, seguido de la disección del tejido, el cual se tomó con pinza Alix para proteger y manipular mejor la muestra de tejido, (Fig. 2) se hace hemostasia con una torunda de gasa estéril, tomando cuatro puntos de sutura con seda $3 / 0$, lavado y secado de la zona. Se dieron indicaciones a la paciente de forma verbal y por escrito.

La paciente regresa a un control posquirúrgico a los 7 días observándose tejidos en buen estado y en proceso de cicatrización. El reporte histopatológico indica mucosa palatina con marcada queratosis sin "displasia".

Se realizan controles mensuales observando la desaparición casi total de la patología; sin embargo la paciente reporta que ha iniciado otra vez el hábito de

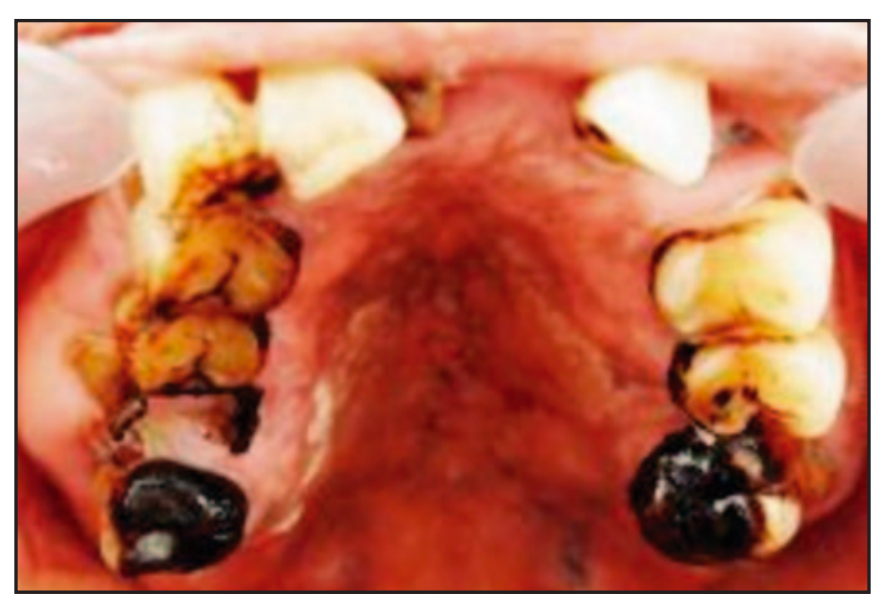

Fig. 2. Imagen fotográfica del maxilar superior donde hay presencia de leucoplasia con disminución de la lesión en tamaño y color.

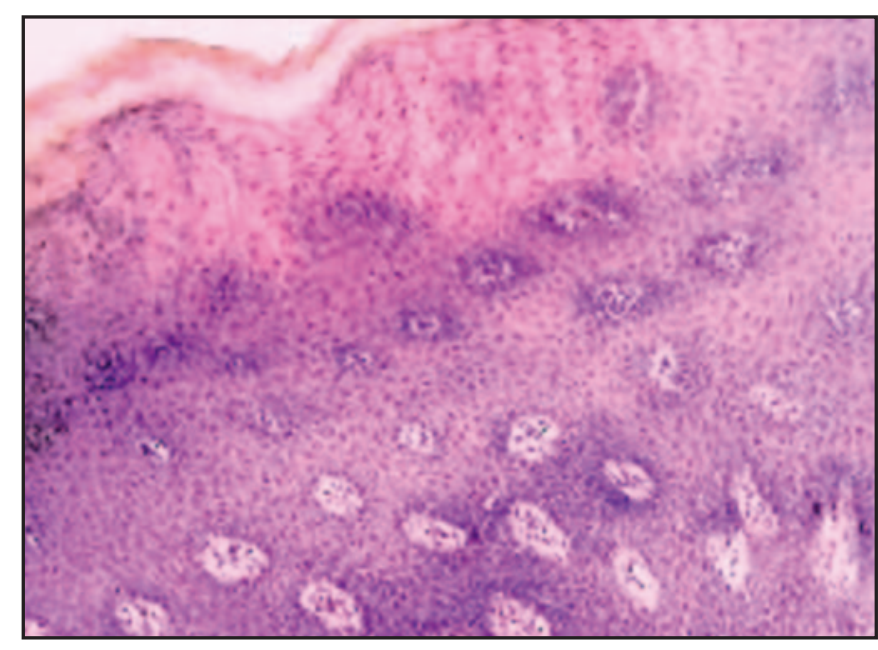

Fig. 3. Fotografía histopatológica donde se observa hiperqueratosis, acantosis e infiltrado inflamatorio crónico.

fumar invertido y, en el último control, se observa aparición nuevamente de las placas blancas. Observamos entonces una fuerte asociación entre el fumar invertido y la aparición de lesiones premalignas.

\section{DISCUSIÓN}

Estudios reportados por Ramulu y col, informan que el 2,4\% del cáncer oral que padecen estos pacientes presentaron la estomatitis nicotínica, ocasionada por fumar de manera invertida, lo que indica que el calor puede ser considerado un agente productor de cáncer (9), lo que coincide con el caso clínico reportado donde la paciente presenta alteraciones en paladar duro como consecuencia del largo periodo de exposición (40 años) por fumar tabaco con la candela invertida.

Autores, como Vellappally, indican: «El tabaquismo es el factor etiológico más importante en el desarrollo de la leucoplasia oral y estudios trasversales reportados muestran una mayor prevalencia de la leucoplasia entre los fumadores, con una relación dosis-respuesta entre el consumo de tabaco y la leucoplasia oral» (4), concordando con el actual caso clínico.

Álvarez G y Álvarez E, en 2008, reportaron, en su estudio realizado en zona rural de municipio del Roble en el departamento de Sucre-Colombia, la alta 
prevalencia de los fumadores invertidos que fue del $15 \%$ en los tres pueblos y el porcentaje más alto se encontró en San Francisco (20\%), menor que el reportado en la India pero mayor que el que se encontró en Colombia en el Tercer Estudio Nacional de Morbilidad, en 1999, que fue aproximadamente el $3,1 \%$. Dicen Álvarez G y Álvarez E que, de las 46 personas clínicamente estudiadas, 42 (91,3\%) eran mujeres (9), lo que coincide con nuestro caso donde se presentó una paciente femenina de 84 años de edad, de raza negra y con el hábito de fumar con la candela invertida por espacio de 40 años.

Gavarasana y Susarla, en 1989 (9), en la India, descubrieron que el hábito de fumar invertido fue un $6,23 \%$ más frecuente en mujeres que en hombres de la sexta década de la vida. Feller y Lemmer demostraron lo contrario en su estudio en África, donde los hombres resultaron más afectados que las mujeres (11); lo que no coincide con el caso clínico reportado (paciente de 84 años).

Para Requena C (2012), la leucoplasia es una lesión que puede tener muchas apariencias clínicas y puede mostrar aspectos histopatológicos muy diferentes. Desde una simple hiperplasia con hiperqueratosis hasta un carcinoma in situ pueden mostrar un aspecto clínico muy similar. Por ello, la biopsia es esencial en los casos persistentes de leucoplasia y, en muchos casos, son recomendables las biopsias repetidas ya que una biopsia con rasgos de benignidad no excluye la evolución premaligna a futuro (12); en nuestro caso, la impresión clínica fue corroborada por estudio anatomopatológico lo que permitió definir con mayor claridad el diagnostico; una biopsia, en este caso, ayudó a la detección temprana de los cambios de la lesión y/o a la prevención de enfermedades malignas como el cáncer oral,

Pindborg, Gupta y Mehta, en 1984, reportaron a través de un estudio que el $48 \%$ de la población investigada mostró cambios en la mucosa palatina asociados con el hábito de fumar de forma convencional; así mismo Álvarez G y Álvarez E concluyeron que en la población de estudio, asociados con el fumar invertido; las lesiones más graves estaban presentes en el paladar, mientras que en la lengua, las lesiones presentaban frecuencia moderadamente alta (9).
En este caso, se muestra que el hábito del tabaquismo de forma inversa sigue utilizándose, hoy por hoy, en poblaciones costeras de América latina donde continúa primando las costumbres o tradiciones en hombres y mujeres; este hábito, practicado con marcada frecuencia, produce lesiones premalignas que pueden convertirse con rapidez en cáncer bucal.

Las poblaciones de escasos recursos económicos son las más vulnerables por el desconocimiento del tabaco como factor de riesgo para la adquisición de enfermedades originadas a partir de los compuestos carcinógenos, contenidos en el humo que afecta no sólo al propio fumador sino a las personas que están a su alrededor inhalándolo, siendo destacable examinar toda la cavidad oral de manera detallada y realizar un buen examen clínico que permita detener o controlar un proceso premaligno.

\section{BIBLIOGRAFÍA}

1. Islam M, Kornberg L, Veenker E, Cohen D, Bhattacharyya I. Anatomic site based ploidy analysis of oral premalignant lesions. Head and neck pathology 2010;4(1):10-4.

2. Van der Wall, Axell T. Oral Leukoplakia: a proposal for uniform reporting. Oral Oncol 2002;38:5216.

3. Amagasa T, Yamashiro M, Uzawa N. Oral premalignant lesions: from a clinical perspective. International Journal of Clinical Oncology 2011;16(1):5-14.

4. Vellappally S, Fiala Z, Smejkalová J, Jacob V, Somanathan R. Smoking related systemic and oral diseases. Acta Medica-Hradec Kralove 2007;50(3):161.

5. Peña González A, Arredondo López M, Vila Martínez L. Comportamiento clínico y epidemiológico del cáncer de cavidad oral. Revista Cubana de Estomatología 2006;43(1):0-0.

6. Harris Ricardo J, Romero Treco J. Leucoplasia homogénea asociada a tabaquismo invertido. Ciencia y Salud Virtual 2012;2(1):161-7. 
7. Hosni ES, Salum FG, Cherubini K, Yurgel LS, Figueiredo Maz. Oral erythroplakia and speckled leukoplakia: retrospective analysis of 13 cases. Revista Brasileira de Otorrinolaringologia 2009;75(2):295-9.

8. Martínez A, Carmona M, Medina E. Comportamiento del cáncer oral en los pacientes atendidos en la unidad de estomatología del Hospital Universitario de Cartagena entre enero de 1991 y diciembre de 1998. Revista colombiana de neumología 2000;12:13-17.

9. Álvarez Gómez GJ, Álvarez Martínez E, Jimenéz Gómez R, Mosquera Silva Y, Gaviria Núñez AM, Garcés Agudelo A, et al. Reverse smokers's and changes in oral mucosa. Department of Sucre, Colombia. Med Oral Patol Oral Cir Bucal 2008 Jan 1;13(1):E1-8.
10. Bascones Martínez A. Medicina Bucal. Tomo I; ed Avances 2009:98-117.

11. Feller, Lemmer J. Oral Leukoplakia as It Relates to HPV Infection: A Review.Hindawi Publishing Corporation International Journal of Dentistry Volume 2012, Article ID 540561, 7 pages doi: 10. 1155/2012/540561.

12. Requena C. Valor diagnostico y pronostico de la histopatología en la leucoplasia oral: Servicio de Dermatología, Instituto Valenciano de Oncología, Valencia, España 2012;27(1):23-6.

\section{CORRESPONDENCIA}

Milse Medina

Milsemagui2@hotmail.com 